\title{
Book review: Zwischen Wildnis und Freizeitpark. Eine Streitschrift zur Zukunft der Alpen
}

\author{
Ernst Steinicke \\ Faculty of Geo and Atmospheric Sciences, University of Innsbruck, Austria \\ Correspondence to: Ernst Steinicke (ernst.steinicke@uibk.ac.at) \\ Published: 5 October 2016
}

Bätzing, Werner: Zwischen Wildnis und Freizeitpark. Eine Streitschrift zur Zukunft der Alpen, Zürich: Rotpunktverlag, ISBN: 978-3-85869-648-9, EUR 10.00, 2015.

Werner Bätzing, geb. 1949 in Kassel, studierter Theologe und emeritierter Professor für Kulturgeographie am Institut für Geographie der Universität Erlangen-Nürnberg, ist es in den vergangenen Jahrzehnten wie kaum einem anderen Gebirgsforscher gelungen, durch seine Publikationen nicht nur in der Fachwelt, sondern auch in der interessierten Öffentlichkeit wahrgenommen zu werden. Er gilt zweifellos als der Alpenkenner. Dies belegt eine Anzahl von Artikeln zu alpenspezifischen Themenkreisen, in besonders eindrucksvoller Weise aber sein Hauptwerk „Die Alpen“. Erstmals 1984 publiziert, erlebte es im Jahr 2015 die vierte Auflage, welche allerdings in völlig überarbeiteter und erweiterter Form mit dem Untertitel „Geschichte und Zukunft einer europäischen Kulturlandschaft" erschienen ist.

Der hier zu besprechende 145 Seiten umfassende kleinformatige Band baut zwar auf diese Neubearbeitung auf, doch bildet er nicht eine Zusammenfassung oder Kurzversion, sondern versteht sich eben als Streitschrift zur Zukunft der Alpen. Das Büchlein stellt in stringenter, sehr verständlicher und - trotz Formats und begrenzter Seitenanzahl - auch in ausführlicher Weise die Hauptaussage dar: Wenn nicht bald eine Trendwende eingeleitet wird, so verschwinden die alpenspezifischen Lebens- und Wirtschaftsformen sukzessive. Dazu bildet der Autor elf Thesen, die in den einzelnen Abschnitten untermauert werden. Da sich die Streitschrift auf das Werk „Die Alpen“ bezieht, sich ausdrücklich an ein breiteres Publikum wendet, ist es durchaus legitim, dass der wissenschaftliche Apparat stark zurücktritt und nur wenige Zitate bereit stellt - all dies im Sinne einer besseren Lesbarkeit.
Die zu Beginn (S. 11-13) im Büchlein genannten Thesen umfassen jeweils den gesamten Alpenbogen; allein schon aus diesem Grund haben räumliche Differenzierungen, welche für die wissenschaftliche Geographie so charakteristisch sind, hier kaum Platz. So geht Bätzing in generalisierender Weise davon aus, dass die Alpen in wirtschaftlicher Hinsicht immer unwichtiger und nur noch eine randliche Rolle als Ergänzungsraum außeralpiner Metropolen einnehmen würden. Vielfältige Kulturlandschaften würden durch Nutzungseinstellung und Verwilderung sowie durch Verstädterung und Zersiedelung verschwinden. Dies beträfe vor allem die tiefen Tallagen, wo sich Bevölkerung und Arbeitsplätze konzentrieren. Der Tourismus sei dagegen nie flächenhaft ausgeprägt gewesen und konzentriere sich auf nur 300 Zentren. Andere größere wirtschaftliche Bereiche in den höheren Lagen, wie die Wasserkraftnutzung, seien kaum arbeitsintensiv; außerdem würde ihr Gewinn aus den Alpen abfließen. Für die Alpen gäbe es somit nur eine Chance, indem sie sich den Problemen der globalen Entwicklung entzieht und die Nutzung der Alpenressourcen in umwelt- und sozialverträglichen Formen stärkt. Gleichzeitig müsse die Bedeutung regionaler Qualitätsprodukte gesteigert werden. Nach Bätzing - und das ist seine letzte These (S. 13) - kann ,diese Aufwertung (...) nicht dadurch umgesetzt werden, dass sich die Alpen nach außen abschotten, sondern nur in Form der Gleichwertigkeit von endogenen und exogenen Nutzungen im Alpenraum - als ausgewogene Doppelnutzung“.

Im ersten anschließenden Hauptkapitel, das sich mit natur-, kultur- bzw. wirtschaftsräumlichen Bereichen beschäftigt und das die „Verwilderung der Alpen“ zum Thema hat, verifiziert der Autor bereits die meisten seiner Thesen. Resümee: Die Alpen zerfallen in verstädterte Gebiete mit anonymen und austauschbaren Strukturen in den Tallagen sowie in Wildnisgebiete in den oberen Stockwerken, wo die traditionellen Ressourcen der Alpen nicht mehr genutzt 
werden und die Verbuschung um sich greift. Bätzing spricht daher insgesamt von ,wildgewordenen Alpen“ (S. 35).

Im folgenden Abschnitt (Kap. II) diskutiert der Autor fünf Zukunftsperspektiven. Die „realistische Perspektive“ stellt den Anschluss der Alpen an die Moderne dar und ließe sich insofern als nachholende Entwicklung interpretieren. Verbesserung der technischen Infrastruktur, Ausweisung von Gewerbeflächen, verstärktes Standortmarketing, Ausweitung des Tourismus in schlecht erreichbaren Gegenden und $\mathrm{Zu}$ sammenarbeit mit außeralpinen Metropolen bilden laut Bätzing diesbezüglich die häufigsten Maßnahmen. Er bewertet diese Entwicklung sehr ablehnend.

Die „neoliberale Perspektive“ wird erwartungsgemäß ebenfalls negativ beurteilt. Bätzing meint, dass sich im kleinstrukturierten Alpenraum wirtschaftliche Erfolge großer Firmen, die im globalen Wettbewerb stehen, kaum erzielen ließen. Standortschließungen seien damit vorprogrammiert. Dem Autor ist vollkommen zuzustimmen, dass dabei die ökologische Dimension - und dies in Zeiten des Klimawandels (Bätzing schreibt auf S. 62 noch „Klimaerwärmung") - außer Acht gelassen wird. Die ,hedonistische Perspektive" sieht die Alpen wiederum als auszubauender Freizeitpark für die europäischen Touristen. Wenig Freude dürften dabei Touristiker mit seiner Geringschätzung von Megasportevents und ihrer Gleichsetzung mit Korruption haben. Bewusst dramatisiert Bätzing, wenn er mit dieser Perspektive in den alpinen Höhenlagen einen Umbau zu einem „Heidi-Idyll“" verbindet. Er sieht auch in dieser Perspektive keine positive Zukunft (S. 65).

Nicht den Freizeitinteressen entspricht das nächste Szenario, das der Autor als „Unterlieger Perspektive“ bezeichnet. Dabei geht es um die Bedeutung der Alpen als Wasserschloss Europas; demnach hätte die wasserwirtschaftliche Nutzung der großen Stauseen Vorrang vor allen anderen Nutzungen (S. 70). Indem Bätzing auf die ökologische Problematik hinweist, lehnt er diese Perspektive genauso ab. Die ,radikal naturschützerische Perspektive“ unterstützt dagegen die Ausbreitung der Wildnis in den Alpen; der Mensch möge sich bewusst zurückziehen, was im Endeffekt eine Abwanderung aus dem Gebirge bedeuten würde. Bätzing bemerkt dazu: „Da diese radikale Naturschutzposition wegen ihrer Selbstwidersprüchlickeit nicht lebbar ist, kann sie für die Alpen keine positive Zukunftsperspektivität bedeuten“" (S. 81).

Gemeinsam sei allen fünf Perspektiven, die Alpen als fremdbestimmten Ergänzungsraum der außeralpinen Metropolen zu betrachten.

Damit leitet Bätzing zum abschließenden dritten Kapitel über, die ,unzeitgemäße Perspektive“, in der er seine „,konkrete, umsetzbare Utopie“ (S. 89) vorstellt. Er fordert zunächst ,kulturelle Werte statt Geld als Schlüsselfaktor“ und meint damit die Aufwertung der alpinen Ressourcen und Potenziale. Konkret werden dabei regionale Qualitätsprodukte angesprochen, wie Getreide, Vieh, Kräuter, Wein, Obst, Steine, Loden u.a. Aus den alpinen Ressourcen mögen die Bewohner (auch Zugezogene und Asylanten) hochwertige, identitätsstiftende Produkte herstellen, wobei die alpine Umwelt stabilisiert bleiben soll (S. 96). „Unzeitgemäß 2“ fordert die Nutzung von dezentralen Ressourcen der Alpen. Die naturräumlichen Bedingungen benachteiligten dort die Intensivierung der Produktion. Das gelte für Landwirtschaft, Bergbau, Forstwirtschaft, Handwerk und Dienstleistungen. Dabei würden allerdings Vorteile der dezentralen Produktion leicht übersehen werden, welche vornehmlich in der hohen Qualität der alpinen Regionalprodukte und der umweltverträglichen Herstellungsweise liegen. Dies bilde eine große Chance für die Alpen als Lebens- und Wirtschaftsraum (S. 106).

Die nächste Forderung betrifft den „Naturschutz mittels angepasster Nutzung", welche den Erhalt der traditionellen Kulturlandschaften vorsieht. Daraus ergeben sich nach Bätzing drei Umweltziele: ,umwelt- und sozialverträgliche Landnutzung“, „Begrenzung des Tourismus“ sowie „Umweltauflagen in den verstädterten Gebieten“ (S. 110f.). „Unzeitgemäß 4“ baut darauf auf: Der Autor nennt diesen Appell selbsterklärend ,multifunktionale Nutzungen statt Monostrukturen“. In dem Zusammenhang greift er die Leitidee der „,ausgewogenen Doppelnutzung“ (S. 120f.) auf: Die endogene Nutzung, also die Nutzung der alpenspezifischen Ressourcen, und die exogene Nutzung (z. Bsp. Wasserkraft oder Metropolfunktionen) mögen in einem ausgewogenen Verhältnis zueinander stehen, wobei die zuletzt Genannte Erstere unterstützen und fördern solle. Das Kapitel „Unzeitgemäß“ wird mit der „Forderung nach alpenspezifischen Lösungen statt globaler Standardisierung" abgeschlossen. Wenn Produkte der globalen Wirtschaft eingeführt werden, so mache sich die Kleinkammerung der Alpen bemerkbar: Standardlösungen würden nicht wirklich passen, alpenspezifische Einzelfalllösungen seien notwendig.

Kapitel III wendet sich daher von der Alternative ab, dass die Alpen entweder Zentrum oder Ergänzungsraum bilden: Mit der Leitidee des multifunktionalen Wirtschaftens und der ausgewogenen Doppelnutzung bringt Bätzing eine neue $\mathrm{Zu}$ kunftsperspektive ein (S. 128). Er schließt die Streitschrift mit dem Gedanken ab, seine Leitidee der ausgewogenen Balance und Gleichwertigkeit von Regionalwirtschaft und globaler Wirtschaft auch für ganz Europa anzuwenden - in zahllosen „Orten guten Lebens“ (S. 135).

Man wäre bei der Lektüre des Bändchens geneigt, Werner Bätzing in allen Punkten rechtzugeben, sind doch seine Leitthesen schwer falsifizierbar. Dennoch ist in einigen Fällen Vorsicht geboten, da die Grundkonzeption davon ausgeht, Feststellungen vornehmen zu können, die für den gesamten Alpenraum gelten. Überzeichnungen sind jedoch in einer Streitschrift durchaus möglich, auch wenn Empirik bzw. landeskundliche Erfahrung nicht immer damit konform gehen: Selbst in den Tallagen der am stärksten zersiedelten Alpengegenden, im Tiroler Inntal, dem Aostatal, Wallis oder in der Furche des Grésivaudan lassen sich zwischen den wachsenden Talorten kilometerlange Spaziergänge in den Auen unternehmen, ohne mit ,wildgewordenen Alpen“ in Kontakt zu geraten. 
Insgesamt werden positive Entwicklungen in den Alpen verschwiegen: So orientiert sich der Autor bei der Wiedergabe von empirischen Daten vor allem an seinem Hauptwerk. Dieses bietet genauere demographische Analysen im Wesentlichen nur für die Gesamtzahl der Einwohnerentwicklung in den Alpengemeinden. Damit übersieht er z.B. wirtschaftlich relevante Migrationen von außeralpinen Gebieten in den Alpenraum. Dass solche Zuwanderungen für die Höhenbereiche in den Alpen durchaus eine Rolle spielen, liegt auf der Hand. Dennoch: Eine verstärkte empirische Analyse könnte zwar die eine oder andere Überspitzung und Dramatisierung gewiss abschwächen, doch müsste man ihm in weiten Teilen zustimmen - selbst wenn man sich schwer tut, so manche These in den slowenischen Berggebieten, im österreichischen Alpenostrand oder in der Montagna friulana nachzuvollziehen.

Andererseits ist Bätzing, der nicht in den Alpen wohnt bzw. auf eine Journalistenfrage offen zugibt, auch nach seiner Emeritierung dort nicht wohnen zu wollen (Tiroler Tageszeitung v. Samstag 2. Mai 2015), dafür bekannt, dass er in seinen Feststellungen ein nicht unbeträchtliches Stück Ideologie und Moral miteinfließen lässt. Nach dem Tourismusforscher Peter Haimayer (2015) fällt dies besonders auf, wenn wachsender Tourismus ein Teil der Verwilderung, stagnierende Bevölkerungszahlen ein positiver Indikator oder Wandern das Allheilmittel gegen die Leere sein soll. So erinnern einige der in der Streitschrift genannten Handlungsempfehlungen mitunter an den Stil längst überholter Kolonialstudien. Oder wie es der Schriftsteller Alois Schöpf in der Leitkolumne derselben Zeitung äußerst provokant formuliert (auch als Reaktion auf die o.g. Journalistenfrage): „Wir aber, die wir in den Alpen wohnen, sollen schon verzichten? Wir sollten wohl zwecks Erhaltung unserer Identitäten für jeden Deutschen leicht nachvollziehbar die Alpenaborigines spielen! Wie seit Jahrhunderten die Dodel vom Land geben! Vielen Dank, Herr Professor! Das kann die Lösung nicht sein!“

http://www.tp-blog.at/allgemeines/

der-alpenraum-auf-talfahrt (aufgerufen im Juni 2015) 